Rev. Biol. Neotrop. 9(1): 19-27. 2012

\title{
IQUEZA DE ESPÉCIES E DIVERSIDADE DE MARCHANTIOPHYTA (HEPÁ- TICAS) DE CAPÕES DE MATA, NO PARQUE NACIONAL DA CHAPADA DOS VEADEIROS, GOIÁS, BRASIL
}

\section{Eliana Marília Lima Pinheiro}

Universidade de Brasília-UnB. Instituto de Ciências Biológicas, Laboratório de Criptógamas, departamento de Botânica. E-mail: biozooeli@yahoo.com.br

Allan Laid Alkimim Faria

Universidade de Brasília-UnB. Instituto de Ciências Biológicas, Laboratório de Criptógamas, departamento de Botânica.

\section{Paulo Eduardo Aguiar Saraiva Câmara}

Universidade de Brasília-UnB. Instituto de Ciências Biológicas, Laboratório de Criptógamas, departamento de Botânica.

\begin{abstract}
Resumo: O Parque Nacional da Chapada dos Veadeiros (PNCV) está localizado ao nordeste do estado de Goiás, e é reconhecido como Patrimônio Mundial Natural pela UNESCO. Na área de campo rupestre do PNCV encontram-se capões de mata, fitofisionomia que proporciona um ambiente favorável ao desenvolvimento de musgos e hepáticas. $\mathrm{O}$ objetivo deste trabalho foi realizar um levantamento das hepáticas associadas aos capões de mata no PNCV, a fim de ampliar o conhecimento sobre a riqueza de espécies e a diversidade, bem como a similaridade entre esses capões. O resultado mostrou uma riqueza específica com 36 espécies, distribuídas em 11 famílias, sendo seis novas ocorrências para o Centro-Oeste e oito novas ocorrências para o estado de Goiás. As famílias mais representativas em quantidade de espécies foram Lejeuneaceae (15 spp.) e Frullaniaceae (cinco spp.), comportando juntas 54\% da riqueza total dos capões. Do total de espécies encontradas, todas ocorrem no bioma Mata Atlântica (100\%); 24 espécies ocorrem na Amazônia (66\%); 16 espécies ocorrem no Pantanal (44\%); oito espécies ocorrem na Caatinga (22\%) e quatro espécies têm ocorrência nos Pampas (11\%). Nenhuma das espécies de hepáticas registradas para os capões de mata consta na Lista Vermelha de espécies ameaçadas da International Union for Conservation of Nature (IUCN).
\end{abstract}

Palavras-Chave: Briófitas, Cerrado, similaridade, formação florestal.

Aвstract: The Chapada dos Veadeiros National Park (PNCV), is located northeast of the state of Goiás, and is recognized as World Heritage by UNESCO. In "campo rupestre" area of PNCV, located a phytophysiognomy classified as "capões de mata" which provides a favorable environment for the development of mosses and liverworts. The objective of this study was to survey of liverworts associated with "capões de mata" in PNCV, in order to increase knowledge of species richness and diversity, as well as the similarity between these "capões". The result showed richness with 36 species belonging to 11 families, six new records for the Centro-Oeste and eight new records for the state of Goiás. The most representative families in number of species were Lejeuneaceae (15 spp.) and Frullaniaceae (five spp.), together comprising $54 \%$ of total richness capões. Of the total number of species, all occurring in the Atlantic Forest biome (100\%), 24 species occur in Amazonia (66\%), 16 species occur in the Pantanal (44\%), eight species occur in the Caatinga (22\%) and four species have occurrence in the Pampas (11\%). None of the species recorded for "capões de mata" consists in IUCN Red List of Threatened Species.

KeY Words: Bryophytes, Cerrado, similarity, forest formation.

\section{INTRODUÇÃO}

A

divisão Marchantiophyta representada pelas hepáticas contém 5.000 espécies. Aliada às divisões Anthocerotophyta (antóceros) e Bryo- phyta (musgos), formam o grupo das briófitas que compreendem cerca de 18.150 espécies distribuídas pelo mundo (Goffinet et al., 2009). Estima-se que existam 1.350 espécies para a região Neotropical (Gradstein et al., 2001) e segundo Costa et al. (2012) o Brasil abriga uma flora de hepáticas composta por 632 espécies. 
A primeira citação sobre as briófitas da região Centro-Oeste consta no trabalho de Sampaio (1916), que catalogou 52 espécies de musgos para o Mato Grosso. Após 35 anos, Hoehne \& Kuhlmann (1951), relataram nove espécies de musgos coletadas para o mesmo estado.

Em 1978 foi publicado o primeiro trabalho de briófitas, especificamente taxonômico, com 44 espécies de musgos para uma região de Floresta Amazônica (Lisboa \& Lisboa, 1978). Em 1985, Guarim-Neto \& Yano (1985), publicaram 26 espécies de briófitas da Serra de São Vicente, região com predominância de vegetação de Cerrado.

Ainda para Mato Grosso, podem-se citar cinco artigos sobre briófitas (Câmara \& Vital, 2004; 2006a; Yano \& Peralta, 2004; 2005; Genevro et al., 2006). Yano \& Peralta (2004; 2005) ampliaram o levantamento das briófitas, com novas ocorrências, sendo seis de musgos e duas de hepáticas. Para Mato Grosso do Sul apenas um artigo (Yano \& Bastos, 2004) com 100 novas ocorrências para o estado.

Os estudos sobre briófitas no estado de Goiás (Yano \& Costa, 2000; Yano \& Peralta, 2007, 2008; Sousa et al., 2008; Peralta et al., 2008) representam uma grande contribuição para o Centro-Oeste. Dentre os artigos Yano \& Peralta (2007; 2008), Peralta et al. (2008) e Sousa et al. (2008) registraram novas ocorrências de briófitas para o estado, sendo 18,67, 40 e 38, respectivamente. Atualmente, o estado de Goiás possui a flora de hepáticas composta por 103 espécies (LISTA DE ESPÉCIES DA FLORA DO BRASIL, 2012).

Para o Distrito Federal pode citar os trabalhos de Filgueiras \& Pereira (1993) - o primeiro para o DF, assim como um Cheklist para o Distrito Federal (Câmara \& Soares 2010); levantamento da brioflora urbana (Câmara et al., 2003), assim como trabalhos em áreas da reserva ecológica do IBGE (RECOR); Águas Emendadas (ESECAE); Jardim Botânico de Brasília; Dolina da Garapa e Parque Nacional de Brasília (Oliveira et al., 2006; Câmara, 2008a; Câmara, 2008b; Carvalho-Silva et al., 2010; Soares \& Oliveira, 2010; Soares et al., 2011).

Apesar de vários trabalhos de levantamentos de briófitas nas regiões do bioma Cerrado, não existem estudos específicos que envolvam a riqueza e a diversidade de hepáticas nos capões de mata. Desta forma, o presente trabalho tem como objetivo realizar um levantamento desse grupo de plantas associadas aos capões de mata no PNCV. Com isso, espera-se ampliar o conhecimento sobre a riqueza de espécies e a diversidade, bem como a similaridade entre os capões.

\section{Material e Métodos}

\section{Área de ESTUdo}

O Parque Nacional da Chapada dos Veadeiros (PNCV) está localizado ao nordeste de Goiás entre as coordenadas geográficas aproximadas $14^{\circ} 03^{\prime} 20^{\prime \prime S}$ e 47030'51"W, com uma área total de 65.514 ha
(Fig. 1). O parque está sob o domínio do Clima Tropical, sub-úmido (AW), segundo Köppen, com duas estações bem definidas: verão chuvoso entre os meses de outubro a abril e inverno seco entre os meses de maio até meados de setembro (Souza \& Felfili, 2006). A precipitação varia de $1.500 \mathrm{~mm}$ a $1750 \mathrm{~mm}$ anuais e a temperatura média anual varia entre $24^{\circ} \mathrm{C}$ e $26^{\circ} \mathrm{C}$ (IBAMA, PROAVES, 1998).

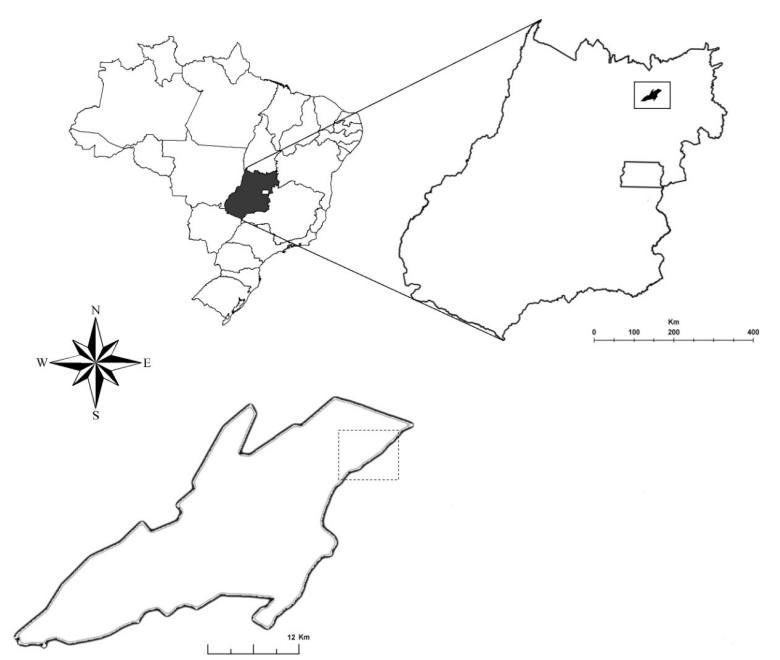

Fig. 1 - Localização do PNCV no Brasil e no estado de Goiás. Abaixo - Área total do PNCV (65.514 ha).

O PNCV é reconhecido como Patrimônio Mundial Natural pela UNESCO e apresenta áreas significativas de sistemas naturais próprios do bioma Cerrado, representadas por formações florestais, savânicas e campestres. A elevada altitude de sua área (em alguns pontos acima de $1.400 \mathrm{~m}$ ) aliada a fatores edáficos e à grande disponibilidade de água contribuem para uma alta diversidade fitofisionômica (ICMBIO/MMA, 2009).

Em meio aos campos rupestres do PNCV encontram-se capões de mata, definidos como manchas de vegetação arbórea de cerrado, cerradão ou mata, formando ilhas nos campos (Magalhães, 1966). Sua ocorrência está associada à inundação ou ao encharcamento sazonal dos solos (Battilani et al., 2011), além de topografia favorável ao acúmulo de sedimentos, nutrientes minerais e orgânicos. Tais fatores associados proporcionam o estabelecimento de espécies florestais nestas manchas (Meguro et al., 1996).

O estudo foi desenvolvido em quatro capões de mata localizados a leste do PNCV (Fig. 2). Os capões foram numerados, sendo que os capões 1,2 e 3 estão localizados a $22 \mathrm{~km}$ ao norte do município de Alto Paraíso, e o capão número 4 localiza-se a $42 \mathrm{~km}$ dos demais, todos no sentido do município de Teresina de Goiás, - Rodovia GO118 , em uma altitude média de $1500 \mathrm{~m}$. 


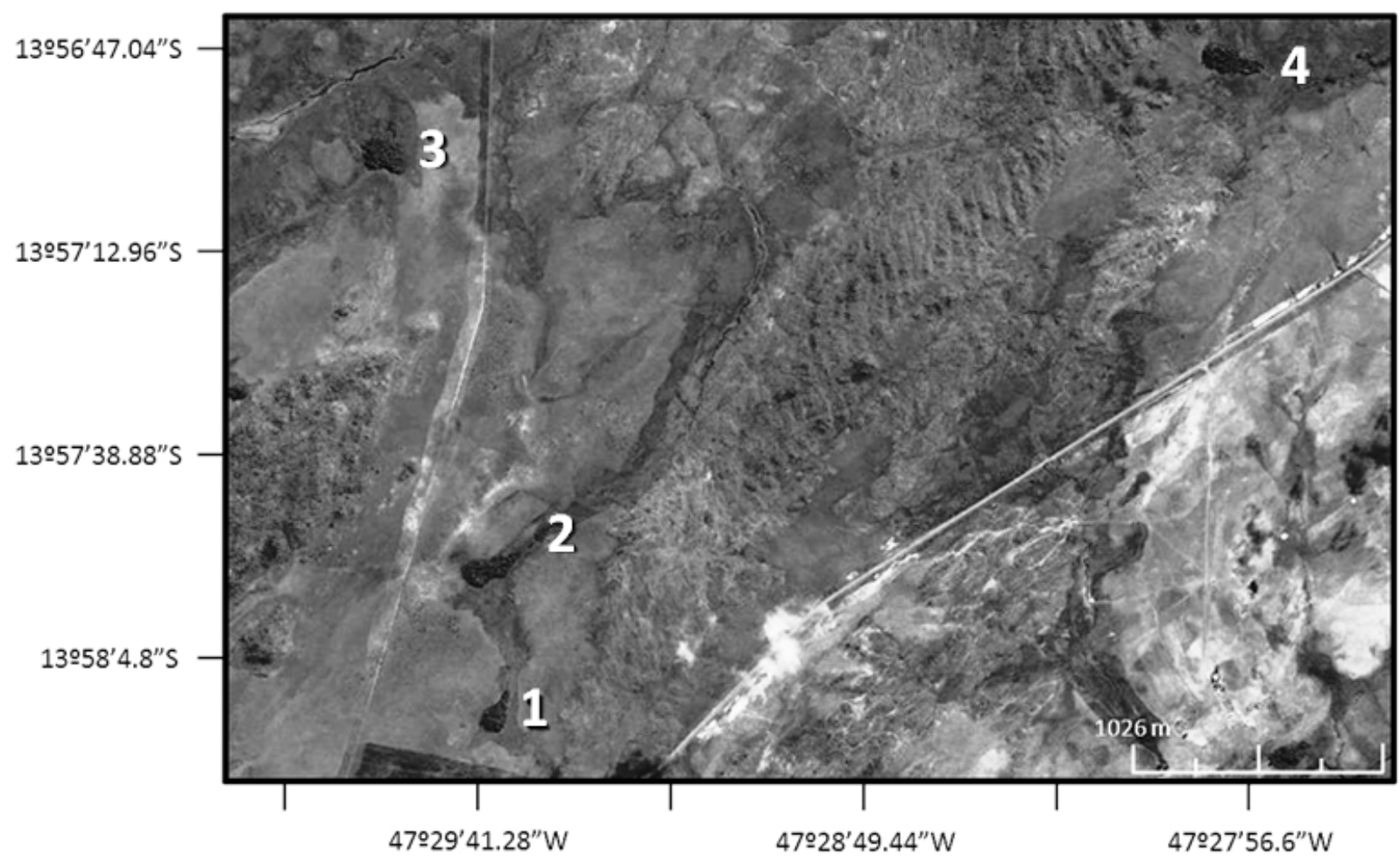

Fig. 2. Vista da área do estudo a partir da imagem de satélite representando os capões 1, 2, 3 e 4 de mata do PNCV. Fonte: Google Earth, acesso: 10/08/2010.

O Capão 1 possui área aproximada de $3.14 \mathrm{Km}^{2}$, entre as coordenadas geográficas: $13^{\circ} 58^{\prime} 12.5^{\prime} \mathrm{S}, 47^{\circ} 29^{\prime} 40.3^{\prime \prime} \mathrm{W}$; e altitude de 1.535 m. É o menor capão em área, sendo o mais próximo da rodovia. Em 2007 este capão foi atingido pelo fogo. No interior do mesmo não há afloramentos d'água. O Capão 2 apresenta área aproximada de $4.52 \mathrm{Km}^{2}$, entre as coordenadas geográficas $13^{\circ} 57^{\prime} 54.4^{\prime \prime} \mathrm{S}, 47^{\circ} 29^{\prime} 42.9^{\prime \prime} \mathrm{W}$, e altitude de $1.520 \mathrm{~m}$. Há um afloramento d'água próximo ao centro do mesmo, seu interior é bastante sombreado e encontra-se a $450 \mathrm{~m}$ de distância do capão 1 .

O Capão 3 possui área aproximada de $3.79 \mathrm{Km}^{2}$, entre as coordenadas geográficas $13057^{\prime} 01.5^{\prime \prime} \mathrm{S}$, 47029"52.4"W; e altitude de $1.475 \mathrm{~m}$. Localizado a $1.5 \mathrm{Km}$ de distância do capão 2 . 0 capão 3 apresenta interior bastante úmido e sombreado, formando áreas pantanosas. 0 capão 4 apresenta área aproximada de $5.30 \mathrm{Km}^{2}$, entre as coordenadas geográficas $13^{\circ} 56^{\prime} 48.1^{\prime \prime} \mathrm{S}$, $47^{\circ} 28^{\prime} 02.6^{\prime \prime} \mathrm{W}$; e altitude de $1.506 \mathrm{~m}$. Está situado atrás de uma formação rochosa, a $2 \mathrm{~km}$ de distância da rodovia. Seu interior é bastante sombreado, há um pequeno córrego, porém sem áreas pantanosas.

\section{Coleta e identificação}

As amostragens foram realizadas aleatoriamente durante a estação seca (agosto/2010) e a estação chuvosa (fevereiro/2011), sendo uma coleta para cada estação. As técnicas de coleta e preservação seguiram a metodologia usual proposta por Frahm (2003).

Para análise do material botânico coletado foram preparadas lâminas permanentes com solução de Hoyer (Anderson, 1954). Posteriormente, através de características morfológicas e morfométricas, o material foi identificado com auxílio de bibliografia especializada, de acordo com Gradstein et al., 2001; Gradstein \& Costa, 2003; Luizi-Ponzo et al,. 2006; Gradstein \& Ilkiu-Borges, 2009. Quando necessário, amostras foram enviadas a especialistas.

O material coletado totalizou 300 amostras que estão depositadas nos herbários da Universidade de Brasília (UB) e do Instituto de Botânica de São Paulo (SP). O sistema de classificação adotado é o de Goffinet et al.(2009) e os dados sobre distribuição geográfica nos biomas foram baseados em Gradstein \& Costa (2003) e em dados online da Lista de Espécies da Flora do Brasil (2012).

\section{ANÁLISE DOS DADOS}

A riqueza específica corresponde ao número de espécies encontrado para cada capão de mata. A partir dos dados de ocorrência das espécies (presença/ausência) entre os quatro capões do PNCV, foram gerados índices através de perfis de diversidades, em que é calculado mais de um índice a fim de explicar qual dos capões é o mais diverso. Para tanto, foram usados os índices de di- 
versidades de Shannon $\left(\mathrm{H}^{\prime}\right)$ e de Simpson. E por último o coeficiente de Jaccard para comparar o grau de similaridade entre os quatro capões analisados. Para obtenção de tais índices foi utilizado o programa PAST 2.15 (Hammer et al., 2001).

\section{Resultados e Discussão}

Foram registradas 36 espécies de hepáticas entre os quatro capões do PNCV na estação chu- vosa, representadas por 11 famílias e 14 gêneros. Para a estação seca, 19 espécies de hepáticas, representadas por nove famílias e 14 gêneros com distribuição para os biomas do Brasil. Do total das espécies, oito são novas ocorrências para o estado de Goiás e seis são novas ocorrências para a região Centro-Oeste, sendo que nenhuma das espécies teve ocorrência exclusiva da estação seca e todas foram registradas na estação chuvosa, porém com maior frequência (Tab.1).

Tab. 1. Marchantiophyta (Hepáticas) dos Capões de Mata do PNCV, Goiás, GO, Brasil. Riqueza representada por famílias e espécie. Ocorrência nos biomas. Estação encontrada. O $\left(^{*}\right)$ representa a primeira ocorrência para o estado de Goiás e $(+)$ representa primeira ocorrência na região Centro-Oeste.

\begin{tabular}{|c|c|c|c|c|c|c|c|}
\hline \multirow[t]{2}{*}{ FAMÍlIA / ESPÉCIE } & \multicolumn{4}{|c|}{ CAPÕES } & \multirow[t]{2}{*}{ OCORRÊNCIA NOS BIOMAS } & \multicolumn{2}{|c|}{ EsTAÇÃo } \\
\hline & 1 & 2 & 3 & 4 & & Seca & Chuv. \\
\hline $\begin{array}{l}\text { ANEURACEAE } \\
\text { Riccardia chamedryfolia (With.) Grolle } \\
\text { CEPHALOZIACEAE }\end{array}$ & & & $x$ & $x$ & Cerrado e Mata Atlântica & $x$ & $x$ \\
\hline $\begin{array}{l}\text { Cephalozia crassifolia (Lindenb. \& Gottsche) Fulford } \\
\text { * Odontoschisma denudatum (Nees) Dumort. }\end{array}$ & $x$ & $\begin{array}{l}x \\
x\end{array}$ & $\begin{array}{l}x \\
x\end{array}$ & $\begin{array}{l}x \\
x\end{array}$ & $\begin{array}{c}\text { Mata Atlântica e Cerrado } \\
\text { Amazônia, Cerrado e Mata } \\
\text { Atlântica }\end{array}$ & $\begin{array}{l}x \\
x\end{array}$ & $\begin{array}{l}x \\
x\end{array}$ \\
\hline Frullania arecae (Spreng.) Gottsche & & $x$ & $x$ & $x$ & Cerrado e Mata Atlântica & & $x$ \\
\hline Frullania brasiliensis Raddi & & $x$ & $x$ & $x$ & $\begin{array}{l}\text { Cerrado e Mata Atlântica } \\
\text { Amazônia, Caatinga, Cerrado, }\end{array}$ & $x$ & $x$ \\
\hline Frullania ericoides (Nees ex Mart.) Mont. & & $x$ & $x$ & $x$ & Mata Atlântica, Pampa e & $x$ & $x$ \\
\hline Frullania kunzei Lehm. \& Lindenb. & $x$ & $x$ & $x$ & $x$ & $\begin{array}{c}\text { Pantanal } \\
\text { Amazônia, Cerrado, Mata } \\
\text { Atlântica e Pantanal }\end{array}$ & $x$ & $x$ \\
\hline Frullania riojaneirensis (Raddi) Spruce & & & $x$ & $x$ & $\begin{array}{l}\text { Amazônia, Cerrado, Mata } \\
\text { Atlântica e Pantanal }\end{array}$ & $x$ & $x$ \\
\hline GEOCALYCACEAE & & & & & & & \\
\hline Chiloscyphus martianus (Nees) Engel \& Schust. & & $x$ & $x$ & $x$ & $\begin{array}{l}\text { Amazônia, Cerrado, Mata } \\
\text { Atlântica e Pantanal }\end{array}$ & $x$ & $x$ \\
\hline JUNGERMANNIACEAE & & & & & & & \\
\hline Jungermannia hyalina Lyell & & $x$ & $x$ & $x$ & $\begin{array}{c}\text { Cerrado, Mata Atlântica e } \\
\text { Pantanal }\end{array}$ & & $x$ \\
\hline $\begin{array}{l}\text { LEJEUNEACEAE } \\
\text { Acanthocoleus aberrans (Lindenb. \& Gottsche) Kruijt }\end{array}$ & & $x$ & $x$ & $x$ & Cerrado, Mata Atlântica e & $x$ & $x$ \\
\hline * + Anoplolejeunea conferta (Meissn. ex Spreng.) Evans & & & $x$ & $x$ & $\begin{array}{c}\text { Pampa } \\
\text { Amazônia e Mata Atlântica }\end{array}$ & $x$ & $x$ \\
\hline * + Aphanolejeunea camillii (Lehm.) R.M. Schust. & & $x$ & $x$ & $x$ & Amazônia, Mata Atlântica & & $x$ \\
\hline * + Brachiolejeunea phyllorhiza (Nees) Kruijt \& Gradst. & & $x$ & $x$ & $x$ & Mata Atlântica & & $x$ \\
\hline Cheilolejeunea clausa (Nees \& Mont.) Stephani & & $x$ & $x$ & $x$ & $\begin{array}{l}\text { Amazônia, Cerrado, Mata } \\
\text { Atlântica e Pantanal }\end{array}$ & & $x$ \\
\hline * + Cheilolejeunea comans (Spruce) Schust. & & $x$ & $x$ & $x$ & Amazônia e Mata Atlântica & & $x$ \\
\hline Cheilolejeunea discoidea (Lehm. \& Lindenb.) Kach. \& Schust. & & $x$ & $x$ & $x$ & Cerrado, Mata Attântica e Pantanal & & $x$ \\
\hline
\end{tabular}


Tab. 1. Marchantiophyta (Hepáticas) dos Capões de Mata do PNCV, Goiás, GO, Brasil. Riqueza representada por famílias e espécie. Ocorrência nos biomas. Estação encontrada. O (*) representa a primeira ocorrência para o estado de Goiás e (+) representa primeira ocorrência na região Centro-Oeste. Continuação.

\begin{tabular}{|c|c|c|c|c|c|c|c|}
\hline \multirow[t]{2}{*}{ FAMÍlia / ESPÉCIE } & \multicolumn{4}{|c|}{ CAPÕES } & \multirow[t]{2}{*}{ OCORRÊNCIA NOS BIOMAS } & \multicolumn{2}{|c|}{ EsTAÇÃo } \\
\hline & 1 & 2 & 3 & 4 & & Seca & Chuv. \\
\hline & & & & & Amazônia, Caatinga, Cerrado, & & \\
\hline Lejeunea flava (Sw.) Nees & $x$ & $x$ & $X$ & $x$ & Mata Atlântica, Pampa e & $X$ & $X$ \\
\hline *Lejeunea glaucescens Gottsche & & $X$ & $X$ & $x$ & $\begin{array}{c}\text { Pantanal } \\
\text { Amazônia, Cerrado, Mata } \\
\text { Atlântica e Pantanal }\end{array}$ & & $X$ \\
\hline Lejeunea laetevirens Nees \& Mont. & & $x$ & $X$ & $x$ & $\begin{array}{c}\text { Amazônia, Caatinga, Cerrado, } \\
\text { Mata Atlântica e Pantanal }\end{array}$ & & $X$ \\
\hline Lejeunea phyllobola Nees \& Mont. ex Mont. & & $x$ & $X$ & $x$ & $\begin{array}{c}\text { Amazônia, Caatinga, Cerrado, } \\
\text { Mata Atlântica e Pantanal }\end{array}$ & & $X$ \\
\hline Lopholejeunea nigricans (Lindenb.) Stephani & & $X$ & $X$ & $x$ & $\begin{array}{c}\text { Amazônia, Cerrado, Mata } \\
\text { Atlântica e Pantanal }\end{array}$ & & $x$ \\
\hline Lopholejeunea subfusca (Nees) Schiffner & & $x$ & $X$ & $X$ & $\begin{array}{l}\text { Amazônia, Caatinga, Cerrado, } \\
\text { Mata Atlântica e Pantanal } \\
\text { Amazônia, Caatinga, Cerrado, }\end{array}$ & & $X$ \\
\hline Microlejeunea bullata (Taylor) Stephani & & $x$ & $X$ & $x$ & Mata Atlântica, Pampa e & $x$ & $x$ \\
\hline Microlejeunea epiphylla Bischle & & $X$ & $X$ & $x$ & $\begin{array}{c}\text { Pantanal } \\
\text { Amazônia, Caatinga, Cerrado, } \\
\text { Mata Atlântica e Pantanal }\end{array}$ & & $x$ \\
\hline LEPIDOZIACEAE & & & & & & & \\
\hline Kurzia capillaris (Sw.) Grolle & & $x$ & $X$ & $X$ & $\begin{array}{c}\text { Amazônia, Cerrado e Mata } \\
\text { Atlântica }\end{array}$ & $x$ & $x$ \\
\hline Kurzia flagellifera (Stephani) Grolle & & $x$ & $X$ & $x$ & $\begin{array}{c}\text { Cerrado e Mata Atlântica } \\
\text { Amazônia, Cerrado e Mata }\end{array}$ & & $x$ \\
\hline Telaranea diacantha (Mont.) Engel \& Merr. & & $X$ & $X$ & $X$ & Atlântica & $X$ & $X$ \\
\hline Telaranea nematodes (Austin) Howe & & $x$ & $X$ & $x$ & $\begin{array}{l}\text { Amazônia, Cerrado e Mata } \\
\text { Atlântica }\end{array}$ & & $x$ \\
\hline LOPHOCOLEACEAE & & & & & & & \\
\hline * + Lophocolea heterophylla (Schrad.) Dumort. & & $X$ & $X$ & $X$ & Cerrado e Mata Atlântica & & $X$ \\
\hline Lophocolea mandonii Stephani & & $X$ & $X$ & $X$ & Cerrado e Mata Atlântica & & $X$ \\
\hline METZGERIACEAE & & & & & & & \\
\hline * Metzgeria ciliata Raddi & $x$ & $x$ & $X$ & $x$ & $\begin{array}{c}\text { Mata Atlântica } \\
\text { Amazônia, Cerrado e Mata }\end{array}$ & $x$ & $x$ \\
\hline Metzgeria furcata (L.) Corda & & $X$ & $X$ & $X$ & Atlântica & $X$ & $X$ \\
\hline Plagiochila corrugata (Nees) Nees \& Mont. & $x$ & $x$ & $X$ & $X$ & $\begin{array}{c}\text { Amazônia, Cerrado e Mata } \\
\text { Atlântica }\end{array}$ & $x$ & $x$ \\
\hline Plagiochila disticha (Lehm. \& Lindenb.) Lindenb. & $x$ & $x$ & $X$ & $x$ & $\begin{array}{c}\text { Amazônia, Caatinga, Cerrado, } \\
\text { Mata Atlântica e Pantanal }\end{array}$ & $x$ & $X$ \\
\hline RADULACEAE & & & & & & & \\
\hline Radula quadrata Gottsche & & $x$ & $X$ & $x$ & $\begin{array}{l}\text { Amazônia e Mata Atlântica e } \\
\text { Cerrado }\end{array}$ & $x$ & $x$ \\
\hline Riqueza específica & 6 & 33 & 36 & 36 & & & \\
\hline
\end{tabular}


As seis espécies, novas ocorrências para - Centro-Oeste são: Aphanolejeunea camillii (Lehm.) Schust. ( $A L, A M, C E, M G, P A, R J$ e $S P$ ), Anoplolejeunea conferta (AL, BA, ES, MG, PA, PB, $\mathrm{PE}, \mathrm{PR}, \mathrm{RJ}, \mathrm{RO}, \mathrm{RS}, \mathrm{SC}$ e SP), Brachiolejeunea phyllorhiza (Nees) Kruijt \& Gradst. (AM, BA, ES, MG, RJ e SP), Cheilolejeunea comans (Spruce) Schust. (AM, BA e SP), Lophocolea heterophylla (Schrad.) Dumort. (PE), Metzgeria ciliata (ES, $M G, P E, P B, R J, R S, S C$ e $S P$ ).

Já as oito espécies de hepáticas ainda não registradas no estado de Goiás são: $A$. camillii, (AL, DF, ES, MG, PR, RJ, RS, SC e SP), A. conferta, B. phyllorhiza, C. comans, L. heterophylla, Lejeunea glaucescens Gottsche (AC, BA, CE, DF, ES, MT, MS, PA, PE, PR, RJ, RR, RS e SP) M. ciliata e Odontoschisma denudatum (Nees) Dumort. (AM, MT, PE, RJ, SC e SP).

Entre as famílias encontradas, nove são hepáticas folhosas (Cephaloziaceae, Frullaniaceae, Geocalycaceae, Jungermanniaceae, Lejeuneaceae, Lepidoziaceae, Lophocoleaceae, Plagiochilaceae e Radulaceae) e apenas duas famílias são hepáticas talosas (Aneuraceae e Metzgeriaceae). As famílias mais representativas em quantidade de espécies foram Lejeuneaceae (15 spp.) e Frullaniaceae (cinco spp.) (Fig. 3), comportando juntas $54 \%$ da riqueza total dos capões.

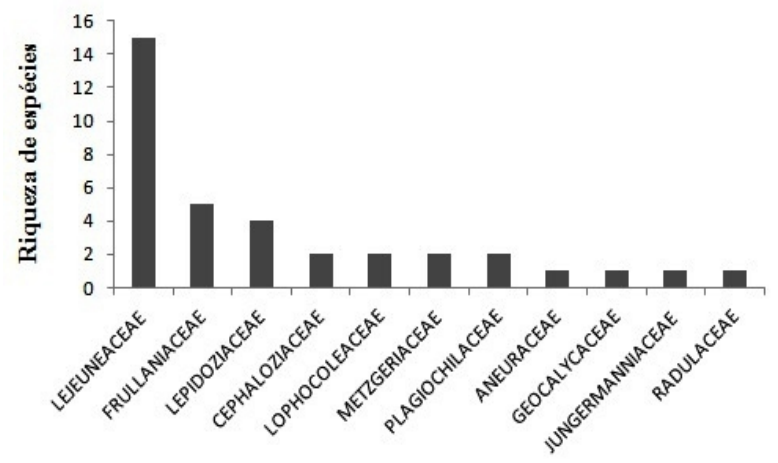

Familias

Fig. 3. Riqueza de espécies representadas por família nos capões de mata do PNCV, Goiás, Brasil.

A grande representatividade de Lejeuneaceae já era esperada, pois essa família predomina na brioflora (Spruce, 1884 ; Gradstein, 1994), como evidenciado nesse trabalho, em que aproximadamente $41 \%$ das espécies levantadas são membros dessa família. Lejeuneaceae também apresentou espécies de primeira ocorrência, sendo cinco novas citações para o estado de Goiás e quatro novas ocorrências para a região Centro-Oeste.

A riqueza de espécies de hepáticas registradas nos capões de mata do PNCV representam 34\% do total de espécies registradas para o estado de Goiás e $22 \%$ do total de espécies para a região Centro-Oeste.

Entre os quatro capões analisados, os que apresentaram maior riqueza específica foram 0 3 e o 4, tanto na estação chuvosa quanto na estaca seca, com 36 espécies nesses dois capões na estação chuvosa e 16 e 14 espécies, respectivamente, na estação seca (Fig.4). A maior riqueza de espécies em tais capões se dá pelo fato da presença de afloramentos de água em seus interiores, o que proporciona microclima úmido, propício para a colonização das hepáticas, principalmente as folhosas.

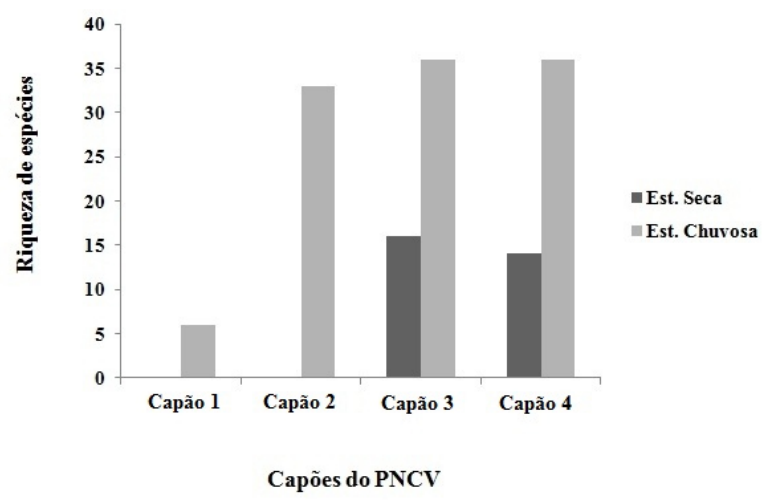

Fig. 4. Riqueza de espécies representadas pelos quatro capões durante as estações seca e chuvosa no PNCV.

A menor ocorrência de espécies no capão 1 (apenas seis espécies em comparação aos demais capões, Tab. 1) pode ser explicada devido à proximidade do mesmo à rodovia, que facilitou o acesso e a antropização; por ter sido atingido pelo fogo, o que tornou os substratos indisponíveis e favoreceu a entrada de maior intensidade luminosa, reduzindo o sombreamento; ao menor tamanho de área em comparação aos demais capões, e principalmente pela ausência de afloramentos de água em seu interior.

A diversidade de hepáticas encontrada nos capões, nas duas estações sazonais (Tab. 2) pode ser explicada pela interação de fatores bióticos e abióticos. Uma grande deciduidade e pouca água disponível no solo constitui importante fator limitante para o crescimento e desenvolvimento das briófitas nos ecossistemas (Bastos, 2008). Isso possivelmente explica a menor diversidade de espécies registrada na estação seca (Tab. 2).

Tab. 2. Índices de diversidades de espécies das comunidades de briófitas entre os quatro capões de Cerrado no PNCV, Goiás, GO, Brasil.

\begin{tabular}{cccccccccc}
\hline $\begin{array}{c}\text { Indice } \\
\text { de diver- } \\
\text { sidade }\end{array}$ & \multicolumn{8}{c}{ Estação / Capão } \\
& \multicolumn{4}{c}{ Seca } & \multicolumn{5}{c}{ Chuvosa } \\
\cline { 2 - 9 } & $\mathbf{1}$ & $\mathbf{2}$ & $\mathbf{3}$ & $\mathbf{4}$ & $\mathbf{1}$ & $\mathbf{2}$ & $\mathbf{3}$ & $\mathbf{4}$ \\
Shannon & 0,693 & 2,633 & 2,649 & 2,619 & 1,801 & 3,076 & 3,402 & 3,416 \\
$\left(\mathrm{H}^{\prime}\right)$ & 0,6 & & & & & & \\
Simpson & 0,500 & 0,917 & 0,917 & 0,916 & 0,815 & 0,936 & 0,961 & 0,962 \\
\hline
\end{tabular}

A similaridade das hepáticas amostradas formou dois grupos principais, segundo o coeficiente de Jaccard (Fig.5). Os capões 3 e 4 tiveram 100\% de similaridade para todas as espécies registradas. Esses capões são formados por vegetação densa e apresentam afloramentos de água e umi- 
dade bastante elevada em seu interior, além de troncos de árvores mortas que favorecem substratos ricos para a formação de diversas comunidades briofíticas. O capão 2 apresentou uma similaridade alta em relação aos capões 3 e 4 com $91 \%$ de similaridade contendo apenas duas espécies: Anoplolejeunea conferta (C. F. W. Meissn. ex Spreng.) A. Evans e Frullania riojaneirensis (Raddi) Spruce, que não foram registradas para os demais capões.

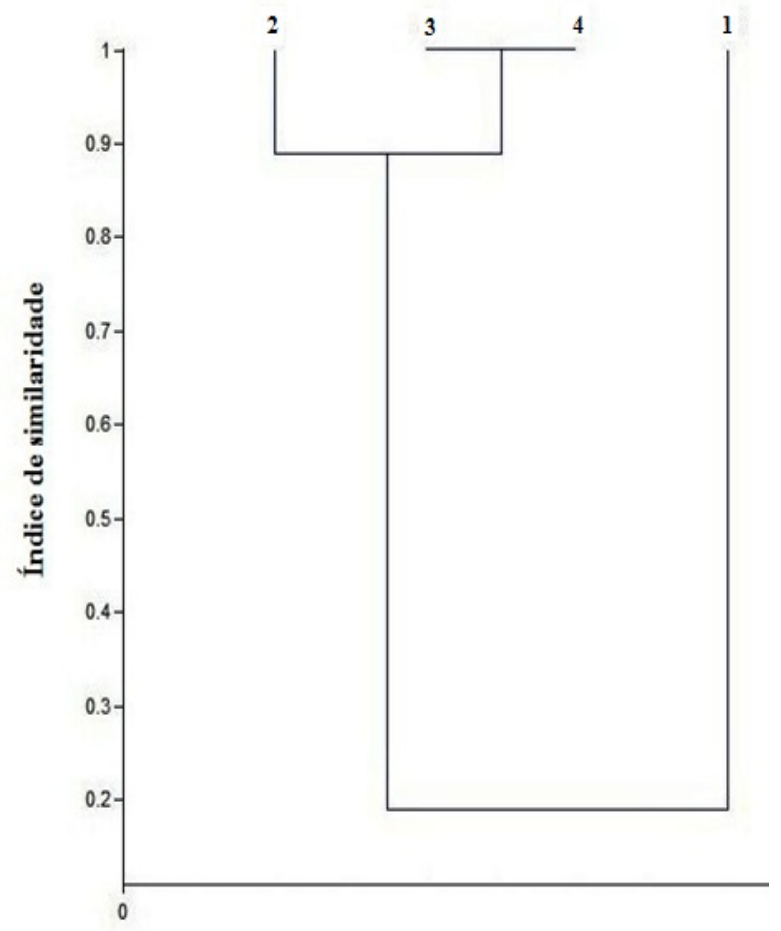

Fig. 5. Similaridade (Coeficiente de Jaccard) entre os quatro capões de mata do PNCV, Goiás, Brasil.

Em relação à distribuição geográfica mundial das hepáticas (Gradstein, 1994; Gradstein \& Costa, 2003), neste trabalho as espécies que ocorrem nos capões de mata do PNCV é representada por $47,6 \%$ de ocorrência, com distribuição na América Tropical (correspondendo a 20 espécies encontradas); $23,8 \%$ de espécies são de distribuição Pantropical (10 espécies analisadas); $12, \%$ de espécies têm distribuição no continente africano (com cinco espécies); 7\% de espécies são Neotropicais (com três espécies), e 4\% são espécies com distribuição cosmopolita e continente europeu, com duas espécies cada.

Quanto à distribuição nos biomas brasileiros, $66 \%$ das espécies provenientes dos capões de mata ocorrem em três ou mais biomas. Do total de espécies encontradas, todas ocorrem no bioma Mata Atlântica (100\%); 24 espécies ocorrem na Amazônia (66\%); 16 espécies ocorrem no Pantanal (44\%); oito espécies ocorrem na Caatinga $(22 \%)$ e quatro espécies têm ocorrência nos Pampas (11\%).

Das espécies encontradas nos capões de mata, $55 \%$ foram também encontradas em tra- balhos de flora no estado do Mato Grosso (Câmara \& Vital, 2004; Câmara e Vital, 2006); 52\% no Parque Estadual da Serra dos Pirineus - Goiás (Sousa et al., 2008), 38\% para o estado do Mato Grosso do Sul (Yano \& Bastos, 2004) e 16\% encontradas nas matas de galeria da reserva ecológica do IBGE no Distrito Federal (Câmara \& Costa, 2006).

Algumas espécies se destacam quanto à ocorrência restrita a determinadas regiões, como os taxa B. phyllorhiza e M. ciliata, registradas apenas para a Mata Atlântica. No Cerrado, os microambientes dos capões de mata (similares às matas de galerias e florestas semi-perenifólias úmidas) são considerados oásis verdes e, junto com os vales, contém a maior diversidade de hepáticas (Gradstein \& Costa, 2003). Além disso, a área dos capões encontra-se em elevada altitude, apresentando temperatura mais amena, e alta disponibilidade de água, o que contribui para a ocorrência de hepáticas em locais úmidos e sombreados.

\section{Conclusão}

Com este estudo, constataram-se novas ocorrências de hepáticas para o estado de Goiás e para a região Centro-Oeste, sendo oito e seis espécies, respectivamente. Dentre estas novas citações, a maior parte delas compõe a família Lejeuneaceae.

Em relação à similaridade de espécies, os capões 3 e 4 apresentaram composição mais similar, visto que possuem condições ambientais bastante semelhantes. $\mathrm{O}$ capão 2 detém uma similaridade de espécies alta em comparação aos capões 3 e 4, no entanto o mesmo apresentou duas espécies que não foram encontradas nos outros capões. O capão 1 apresentou uma riqueza de espécies extremamente baixa em comparação aos demais capões, possivelmente devido à baixa disponibilidade de água, tamanho de área e antropização.

Nesse levantamento, nenhuma das espécies de hepáticas dos capões de mata consta na Lista Vermelha de espécies ameaçadas da IUCN (International Union for Conservation of Nature). Portanto, além das áreas do PNCV assegurarem a manutenção de amostras representativas de ambientes naturais próprios do Cerrado, garantem a conservação das espécies de hepáticas.

\section{Agradecimentos}

Agradecemos aos especialistas que confirmaram algumas das espécies: Dra. Denise P. Costa, Dr. Denilson Peralta e Dr. Cid Bastos. Aos coletores Walfrido Salmito, Msc. Abel Soares, Msc. Bruno Edson. Ao orientador Dr. Paulo Câmara e Dra. Micheline Carvalho pelo apoio e incentivo. 


\section{REFERÊNCIAS}

Anderson, L.E. 1954. Hoyer's solution as a rapid permanent mounting medium for bryologists. Bryologist 57: 242-244.

Bastos, C.J.P. 2008. Padrões de reprodução vegetativa em espécies de Lejeuneaceae (Marchantiophyta) e seu significado taxonômico e ecológico. Rev. Bras. Bot. 31: 309-315.

Battilani, J.L., C.M.M. Ferreira, N.C. Penatti, C. Viadoto, G.A. Damasceno-Júnior \& P. Arnildo. 2011. Análise Comparativa da Estrutura de Capões de Floresta Estacional Semidecídua na Sub-Região do Pantanal de Miranda, MS, Brasil, com Diferentes Métodos de Amostragem, p. 520-536. In: J.M. Felfili, P.V. Eisenlohr, M.M.R.F. Melo, L.A. Andrade \& J.A.A.M. Neto (Ed.) Fitossociologia no Brasil - Métodos e estudos de casos. Universidade Federal de Viçosa, UFV. Viçosa, MG.

Câmara, P.E.A.S. \& D.M. Vital. 2004. Briófitas do município de Poconé, Pantanal de Mato Grosso, MT, Brasil. Acta bot. Bras. 18: 881-886.

Câmara, P.E.A.S., J.R.P.M. Oliveira \& M.M.M. Santiago. 2005. A Checklist of the bryophytes of Distrito Federal (Brasília, Brazil). Trop. Bryol. 26: 133-140.

Câmara, P.E.A.S. \& D.M. Vital. 2006. Briófitos de Cáceres, Pantanal de Matogrosso, Brasil, con nuevos registros para el estado y el Pais. Trop. Bryol. 27: 1-8.

Câmara, P.E.A.S. \& D.P. Costa. 2006. Hepáticas e antóceros das matas de galeria da Reserva Ecológica do IBGE, RECOR, Distrito Federal. Hoehnea 33: 79:87.

Câmara, P.E.A.S. 2008. Musgos pleurocárpicos das matas de galeria da Reserva Ecológica do IBGE, RECOR, Distrito Federal, Brasil. Acta bot. bras. 22(2): 573-581.

Câmara, P.E.A.S. 2008. Musgos acrocárpicos das matas de galeria da Reserva Ecológica do IBGE, RECOR, Distrito Federal, Brasil. Acta Bot. Bras. 22: 1027-1035.

Câmara, P.E.A.S. \& A.E.R. Soares. 2010. A new and update bryophyte checklist for Distrito Federal (Brasília, Brazil). Trop. Bryol. 31: 171-174.

Carvalho-Silva, M., A.E.R. Soares, P.E.A.S. Câmara \& R.G.D. Neto. 2010. Levantamento de musgos (Bryophyta) do Jardim Botânico de Brasília, Distrito Federal, Brasil. Heringeriana 4: 11-27.
Costa, D.P., D.F. Peralta \& N.D. Santos. 2012. Hepáticas in Lista de Espécies da Flora do Brasil. Jardim Botânico do Rio de Janeiro. (http:// floradobrasil.jbrj.gov.br/2012/FB000011).

Filgueiras, T.S. \& B.A.S. Pereira. 1993. Flora do Distrito Federal In: M.N Pinto (org.) Cerrado: caracterização, ocupação e perspectivas. 2 ed. Brasília, Ed. Universidade de Brasília.

Frahm, J.P. 2003. Manual of tropical bryology. Trop. Bryol. 23: 5-195.

Genevro, J.A., F.P. Filho \& D.F. Peralta. 2006. Briófitas das matas de galeria no Parque Municipal Mário Viana, Nova Xavantina, Mato Grosso, Brasil. Bol. do Inst. de Bot. (São Paulo) 18: 149-157.

Goffinet, B., W.R. Buck \& A.J. Shaw. 2009. Morphology and classification of the Bryophyta. P. 55-138. In: Goffinet, B. \& A.J. Shaw (Eds.) Bryophyte Biology. University Press, Cambridge, Havard.

Gradstein, S.R. 1994. Lejeuneaceae: Ptychantheae, Brachiolejeuneae. Flora Neotrop. Monogr. 62: 1-216.

Gradstein S.R., S. Churchil \& N. Salazar-Allen. 2001. A guide to the bryophytes of tropical America. Mem. New York Bot. Gard. V. 86.

Gradstein, S.R. \& D.P. Costa. 2003. The hepaticae and anthocerotae of Brazil. Mem. New York Bot. Gard. V. 87.

Gradstein, S.R. \& A.L. Ilkiu-Borges. 2009. Guide to the plants of Central French Guiana. Mem. New York Bot. Gard. V. 76, part 4.

Guarim-Neto, G. \& O. Yano. 1985. Brioflora da Serra de São Vicente, Mato Grosso. Rev. Bras. Bot. 8: 199-202.

Hammer, O., D.A.T. Harpper \& P.D. Ryan. 2001. PAST: Paleontological Statistics software package for education and data analysis. Palaentol. Electronica 4: 9.

Hoehne, F.C. \& J.G. KuhImann. 1951. Índice bibliográfico e numérico das plantas colhidas pela Comissão Rondon ou Comissão de linhas telegráficas, estratégicas de Mato Grosso ao Amazonas, de 1908 até 1923 . Secretaria da Agricultura, São Paulo.

IBAMA / PROAVES. 1998. Parque Nacional da Chapada dos Veadeiros: Plano de manejo Fase 3 (versão preliminar). Brasília: Ibama / Proaves.

ICMBIO/MMA. 2009. Plano de Manejo do Parque Nacional da Chapada dos Veadeiros. Brasília: Ministério do Meio Ambiente. 
Lisboa, R.C.L. \& P.L.B. Lisboa. 1978. Contribuição ao conhecimento da flora do Aripuanã (Mato Grosso) II. Acta Amaz. 8: 143-148.

Lista de Espécies da Flora do Brasil. 2012 in http://floradobrasil.jbrj.gov.br/2012 (Acesso em 04/09/2012).

Luizi-Ponzo, A.P, C.J.P. Bastos, D.P. Costa, P.E.A.S. Câmara, K.C. Porto, R.C.L. Lisboa \& S. Boas-Bastos. 2006. Glossarium Polyglottum Bryologiae - Versão Brasileira do Glossário Briológico. Editora UFJF, Juiz de Fora.

Magalhães, G.M. 1966. Sobre os cerrados de Minas Gerais. Ana. Acad. Bras. Ciênc. 38: 59-69.

Meguro, M., J.R. Pirani, R. Mello-Silva \& A.M. Giulietti. 1996b. Caracterização florística e estrutural de matas ripárias e capões de altitude da Serra do Cipó, Minas Gerais. Bol. Bot. Univ. São Paulo 15: 13-29.

Oliveira, J.R.P.M., L.D.P. Alvarenga \& K.C. Porto. 2006. Briófitas da Estação Ecológica de Águas Emendadas, Distrito Federal, material coletado por Daniel Moreira Vital. Bol. do Inst. de Bot. (São Paulo) 18: 181-195.

Peralta, D.F., J. Bordin \& O.Yano. 2008. Novas ocorrências de musgos (Bryophyta) para os Estados de Goiás e Tocantins. Acta Bot. Bras. 22: 834-844.

Soares, A.R. \& J.R.P.M. Oliveira. 2010. Briófitas da Dolina da Garapa, Apa de Cafuringa, Distrito Federal, Brasil. Heringeriana 4: 56-66.

Soares, A.E.R., P.E.A.S. Câmara \& D.F. Peralta. 2011. Mosses of gallery forests from Brasilia National Park. Bol. do Inst. de Bot. (São Paulo) 21: 185-192.
Sousa, M.A.R., V.L.G. Klein, M.H. Rezende \& O. Yano. 2008. Antóceros e hepáticas do Parque Estadual da Serra dos Pirineus e arredores, Município de Pirenópolis, Goiás, Brasil. Rev. Biol. Neotro. 5: 1-16.

Souza, C.D. \& J.M. Felfili. 2006. Uso de plantas medicinais na região de Alto Paraíso de Goiás, GO, Brasil. Acta Bot. Bras. 20: 135-142.

Spruce, R.M. 1884. Hepaticae Amazonicae et Andinae. Transactions and Proccedings of the Botanical Society Edinburg 15: 1-588.

Yano, O. \& D.P.Costa. 2000. Criptógamos: Briófitas, p. 1-33. In: J. A. Rizzo (Coord), Flora dos estados de Goiás e Tocantins. Goiânia, PRPPG/UFG.

Yano O. \& C.J.P. Bastos. 2004. Adições à flora de Briófitas de Mato Grosso do Sul, Brasil. Acta Bot. Bras. 18: 437-458.

Yano, O. \& D.F. Peralta. 2004. Musgos (Bryophyta) de Mato Grosso, Brasil. Hoehnea 31: 251-292.

Yano, O. \& D.F. Peralta. 2005. Hepáticas (Marchantiophyta) de Mato Grosso. Hoehnea 32: 185-205.

Yano, O. \& D.F. Peralta. 2007. Criptógamos: Briófitas, p. 1-333. In: J.A. Rizzo (Coord.) Flora dos Estados de Goiás e Tocantins. Goiânia, PRPPG/UFG.

Yano, O. \& D.F. Peralta. 2008. Criptógamos: Antóceros (Anthocerotophyta) e Hepáticas (Marchantiophyta), p. 1-277. In: J.A. Rizzo (Coord.) Flora dos Estados de Goiás e Tocantins. Goiânia, PRPPG/UFG.

Recebido em 13.IX.2012 Aceito em 06.II.2013 